\title{
ФИЛЬТРАЦИЯ ЖИДКОСТЕЙ В АНОМАЛЬНЫХ КОЛЛЕКТОРАХ
}

Грачев Сергей Иванович', grachevsi@mail.ru

\author{
Коротенко Валентин Алексеевич', \\ grachevsi@mail.ru
}

\section{Кушакова Нелли Петровна', kushakovanp@tyuiu.ru}

\author{
Кряквин Александр Борисович', \\ grachevsi@mail.ru
}

\author{
Зотова Ольга Павловна', \\ zotovaop@tsogu.ru \\ 1 Тюменский индустриальный университет, \\ Россия, 625000, г. Тюмень, ул. Володарского, 38.
}

\begin{abstract}
Актуальность исследования обусловлена необходимостью разработки эксплуатационных объектов юрских и ачимовских отложений с низкими фильтрационными свойствами и залежей вязкопластичной нефти. Для исследования фильтрации флюидов в указанных коллекторах нельзя применять классический закон Дарси. Поэтому для прогнозирования технологических показателей разработки и расчёта добывных возможностей скважин следует учитывать возникающие особенности процессов фильтрации и вытеснения нефти.

Цель: установление связи между параметром Лагранжа и начальным градиентом давления; определение значений параметра Лагранжа при нарушении пределов применимости закона Дарси; обоснование применения методов гидродинамического моделирования, разработанных для описания фильтрационных процессов вязкопластичной жидкости, к изучению движения флюидов в низкопроницаемых коллекторах.

Объекты: высокопроницаемые коллектора, насыщенные высоковязкой (вязкопластичной) нефтью; низкопроницаемые пласты ачимовских и юрских отложений.

Методы: интерпретация результатов лабораторных исследований керна; физическое моделирование фильтрационных процессов флюидов в аномальных коллекторах.

Результаты. Отклонение от закона Дарси при фильтрации жидкостей в аномальных коллекторах определяется посредством вычисления значений параметра Лагранжа. Предложен способ определения нижней границы применимости закона Дарси, при нарушении которого для описания предлагаются две модели: с начальным градиентом давления и нелинейная модель. Для процессов фильтрации с нарушением верхнего предела применимости закона Дарси рассмотрена нелинейная модель. Приведены примеры определения параметра Лагранжа для случаев нарушения нижней и верхней границ закона Дарси. Для описания процессов фильтрации дилатантных и псевдопластических жидкостей вместо нелинейных уравнений предлагается использовать систему уравнений пьезопроводности. Показано, что методы решения гидродинамических задач фильтрации и вытеснения нефти, установленные для вязкопластичных нефтей, применимы и для низкопроницаемых коллекторов.
\end{abstract}

\section{Ключевые слова:}

Аномальные коллектора, пределы применимости закона Дарси, начальный градиент давления, коэффициент подвижности, параметр Лагранжа.

\section{Введение}

Под аномальными, или нетрадиционными, коллекторами будем понимать пласты, фильтрация в которых не подчиняется классическому закону Дарси. К ним относятся высокопроницаемые отложения, насыщенные высоковязкой нефтью (BBH) а также пласты, насыщенные маловязкой нефтью, но обладающие низкими фильтрационными свойствами. Вопросам установления верхнего и нижнего пределов применимости закона Дарси посвящено большое количество исследований как у нас в стране [1-19], так и за рубежом [20-37]. Отклонения от верхнего и нижнего пределов фильтрации применимости закона Дарси определяются числом Рейнольдса, параметром Дарси, введенного В.Н. Щелкачевым, параметром Лагранжа. Пре- вышение верхнего предела Дарси имеет место в высокопроницаемых коллекторах. При высоких значениях коэффициента проницаемости, что соответствует большим диаметрам поровых каналов, количество частиц жидкости, взаимодействующих со стенками поровых высокопроницаемых каналов, гораздо меньше, чем количество частиц флюида, участвующих в фильтрации. Уравнение движения (нелинейный закон Дарси), описывающее фильтрацию, содержит слагаемое, зависящее от квадрата скорости фильтрации.

Для анализа процессов фильтрации ВВН А.Т. Горбуновым [1] использован коэффициент подвижности. А.Х. Мирзаджанзаде [2] предложил при исследовании фильтрации вязкопластичных нефтей (ВПН) учитывать начальное касательное 
напряжение, широко используемое при решении задач подземной гидродинамики, которое в дальнейших работах А.Х. Мирзаджанзаде и других авторов было заменено начальным градиентом давления, широко используемое при решении задач подземной гидродинамики.

\section{Определение нижней границы \\ применимости закона Дарси}

В настоящей работе предложен способ определения нижней границы применимости закона Дарси и рассмотрены особенности фильтрации флюидов в аномальных коллекторах.

Для определения нижней границы примененимости закона Дарси авторами предлагается воспользоваться коэффициентом подвижности $k / \mu$, где $k$ - коэффициент проницаемости; $\mu$ - коэффициент динамической вязкости. Для расчетов использовались данные исследований по подсчёту запасов месторождений Тюменской области (Ершовое, Хохряковское, Русское и другие), проведённых в ЗапСибНИГНИ при участии авторов настоящей статьи. Результаты расчётов коэффициентов подвижности для разных значений $k$ и $\mu$ приведены в табл. 1.

Таблица 1. Определение нижней гранииы применимости закона Дарси

Table 1. Definition of a lower limit of applicability of Darcy's law

\begin{tabular}{|c|c|c|c|c|}
\hline $\begin{array}{c}k, \mathrm{~mL} \\
\mathrm{mD}\end{array}$ & $\begin{array}{c}\mu, \text { мПас } \\
\text { mPA.s }\end{array}$ & $\begin{array}{c}k / \mu, \mathrm{m} / \mathrm{m} \Pi а \mathrm{ac} \\
\mathrm{mD} / \mathrm{mPA} \mathrm{s}\end{array}$ & \begin{tabular}{|c|} 
Закон Дарси \\
Darcy's law
\end{tabular} & $\begin{array}{l}\text { Тип коллектора } \\
\text { Collector type }\end{array}$ \\
\hline 100 & 5 & 20 & \multirow{4}{*}{$\begin{array}{l}\text { классичес- } \\
\text { кий } \\
\text { classical }\end{array}$} & \multirow{4}{*}{$\begin{array}{c}\text { обычный } \\
\text { normal }\end{array}$} \\
\hline 200 & 4 & 50 & & \\
\hline 20 & 2 & 10 & & \\
\hline 10 & 1 & 10 & & \\
\hline 10 & 2 & 5 & \multirow{5}{*}{$\begin{array}{c}\text { обобщенный } \\
\text { generalized }\end{array}$} & \multirow{3}{*}{\begin{tabular}{|c|} 
аномальный, \\
низкопроницаемый \\
anomalous, \\
low-permeability
\end{tabular}} \\
\hline 5 & 1 & 5 & & \\
\hline 4 & 1 & 4 & & \\
\hline 200 & 200 & 1 & & \multirow{2}{*}{$\begin{array}{c}\text { аномальный, с ВПН } \\
\text { anomalous, } \\
\text { viscoplastic oil }\end{array}$} \\
\hline 500 & 300 & 1,67 & & \\
\hline
\end{tabular}

В первых четырех строках приведены значения коэффициентов подвижности для обычных коллекторов, в которых процессы фильтрации подчиняются закону Дарси. В строках 5-7 рассчитан коэффициент подвижности низкопроницаемых коллекторов маловязкой нефти. В последних строках приведены значения коэффициентов подвижности для коллекторов с ВПН. Для аномальных коллекторов значения коэффициента подвижности $\leq 10 \mathrm{мДа/мПас.} \mathrm{Потому} \mathrm{величину} \mathrm{коэффициента}$ подвижности, равную $10 \mathrm{мДа/мПас}=10^{-11} \mathrm{Mkм}^{2} /$ Пас предлагается принять в качестве нижнего предела применимости закона Дарси.

Заметим, что, как и в каждой классификации, посвященной определению особенностей строения физического (природного) объекта, численные параметры - вещь весьма условная. Дальнейшие экспериментальные исследования могут изменить выбранную границу. Так, для оценки значений коэффициента подвижности следует учитывать состав и структуру нефтей, строение и литологию коллектора. Поэтому если учитывать, что состав нефти изменяется в процессе разработки и литологические особенности строения коллектора, то в общем случае нижний предел применимости закона Дарси будет функцией нескольких переменных.

\section{Зависимость между параметром Лагранжа} и начальным градиентом давления

В монографии А.П. Телкова, С.И. Грачева [13] отклонение от классического закона Дарси предлагается учитывать параметром Лагранжа $\lambda^{*}$, зависящим от числа Рейнольдса Re и параметров пористой среды. Скорость фильтрации в этом случае равна

$$
v=\frac{1}{\lambda^{*}(\operatorname{Re})} \frac{k}{\mu} \frac{\partial p}{\partial x},
$$

где $\partial p / \partial x-$ градиент давления.

При $\lambda^{*}=1$ выполняется закон Дарси, при величине $\lambda^{*}>1$ нарушается нижний предел применимости закона Дарси, если $\lambda^{*}<1$ нарушается верхний предел применимости закона Дарси. Заметим, что формула (1) выполняется для постоянного коэффициента подвижности. Если скорость фильтрации постоянна, что имеет место при обработке лабораторных экспериментов, то после интегрирования (1) получим, что параметр Лагранжа является обратной величиной параметра Дарси

$$
\lambda^{*}=\frac{k \Delta p}{\mu v l}=\frac{k F \Delta p}{\mu Q l}=\frac{1}{\not \grave{a}} .
$$

Здесь $v$ - скорость фильтрации; $l$ - линейный размер образца или граница области прямолинейно-параллельной фильтрации; $Q$ - дебит или расход; $F$ - площадь фильтрации; $\Delta p$ - перепад давления.

Чтобы применить параметр Лагранжа для анализа промысловых данных обобщим методику определения нарушения закона Дарси, рассмотренную в [13], для стационарной плоскорадиальной фильтрации. Выразим скорость фильтрации через дебит, по аналогии с (1)

$$
v=\frac{Q}{2 \pi r h}=\frac{k}{\lambda^{*} \mu} \frac{\partial p}{\partial r} .
$$

После интегрирования получим выражение, аналогичное формуле Дюпюи

$$
Q=\frac{2 \pi k h \Delta p}{\mu \lambda^{*} \operatorname{Ln} \frac{R}{r_{c}}} .
$$

Здесь $h$ - толщина пласта; $R$ - радиус контура питания; $r_{c}$ - радиус скважины.

Из (3) следует, что при параметре Лагранжа $\lambda^{*}$ отличного от 1 имеет место нарушение закона Дарси. Зависимость $Q$ от $\Delta p$ изображена на рис. 1. 


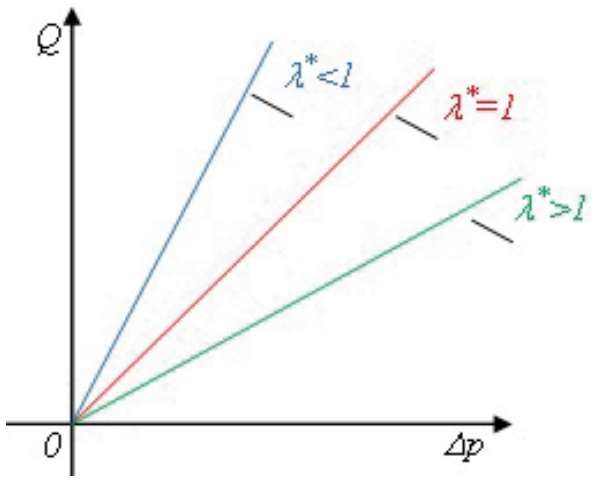

Рис. 1. Зависимость дебита от депрессии для различных значений параметра Лагранжа $\lambda$ *

Fig. 1. Flow rate dependence on depression for various values of the parameter of Lagrange $\lambda^{*}$

При одной и той же депрессии для $\lambda^{*}>1$ получим меньший дебит, а для $\lambda^{*}<1$ дебит больше, чем при выполнении закона Дарси. В общем случае линии рис. 1 , соответствующие $\lambda^{*}>1$ и $\lambda^{*}<1$, не являются прямыми линиями.

Установим связь между параметром Лагранжа и начальным градиентом давления. Скорость фильтрации подчиняется обобщенному закону Дарси:

$$
\vec{v}=-\frac{k}{\mu}(\operatorname{grad} p-\vec{g}),
$$

где $g$ - начальный градиент давления, gradp - текущий градиент давления. При gradp $\leq$ g скорость фильтрации $v=0$. Если $g=0$, то выполняется классический закон Дарси.

Для установления связи между начальным градиентом давления и параметром Лагранжа проинтегрируем уравнение пьезопроводности стационарной фильтрации для обобщенного закона Дарси (4)

$$
\frac{\partial}{\partial r}\left[\frac{\partial}{\partial r} r\left(\frac{\partial p}{\partial r}-g\right)\right]=0 .
$$

Дебит скважины в этом случае

$$
Q=\frac{2 \pi k h}{\mu} \frac{\left(\Delta p-\Delta p^{*}\right)}{\operatorname{Ln} \frac{R}{r_{c}}}, \Delta p>\Delta p^{*} .
$$

Приравнивая (3) и (5), получим

$$
\lambda^{*}=\frac{1}{1-\frac{\Delta p^{*}}{\Delta p}}>1 \text {. }
$$

Способ определения начального градиента давления для ВПН на установившихся режимах фильтрации предложен в работах авторов [12, 38].

$$
g=\frac{\Delta p^{*}}{R-r_{c}-r_{c} \operatorname{Ln} \frac{R}{r_{c}}},
$$

здесь $\Delta p^{*}$ - предельное значение депрессии, соответствующее началу фильтрации; $R$ - радиус кон- тура питания; $r_{c}$ - радиус скважины. Формулы (6) и (7) устанавливают связь между начальным градиентом давления и параметром Лагранжа.

Таким образом, нарушение нижней границы фильтрации закона Дарси можно определить по результатам интерпретации индикаторных диаграмм.

Уравнения пьезопроводности

\section{при нарушении применимости закона Дарси}

Нарушение закона Дарси имеет место не только при фильтрации ВПН и маловязких флюидов в низкопроницаемых коллекторах, но и при течении флюидов с особыми структурно-механическими свойствами в коллекторах большой проницаемости. По результатам лабораторных исследований реологических свойств жидкостей, обладающих особыми структурно-механическими свойствами, выделены три группы: вязкопластические (вязкопластичные) нефти, псевдопластичные флюиды (псевдопластики) и дилатантные жидкости. К последним относятся: водные растворы полимеров (например, полиакриломида), а также жидкости, применяемые для выравнивания профиля притока. На рис. 2 изображены зависимости касательных напряжений от скорости деформации сдвига [4].

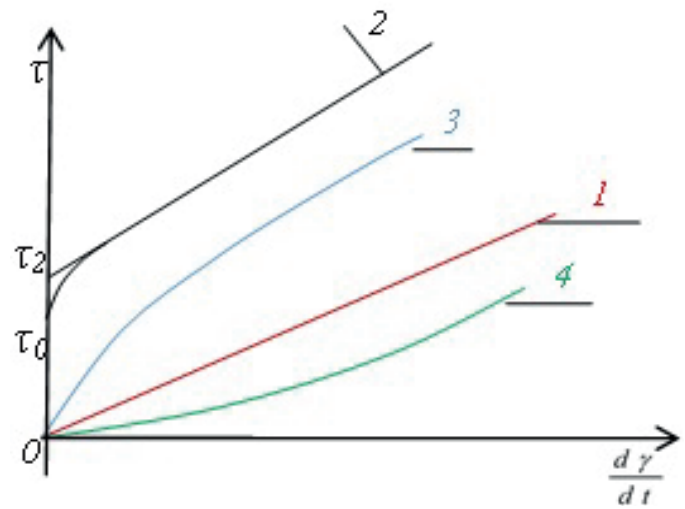

Рис. 2. Зависимость касательных напряжений от скоростей деформаиии сдвига высоковязких нефтей. 1 - ньютоновская жидкость; 2 - вязкопластическая жидкость (ВПЖ); 3 - псевдопластики; 4 - дилатантная жидкость; $\tau$ - напряжение сдвига; $\frac{d \gamma}{d t}-$ скорость деформаиии сдвига; $\tau_{0}$ - начальное касательное напряжение для ВПЖ; $\tau_{2}$-приближенное значение начального касательного напряжения для ВПЖ

Fig. 2. Dependence of shear stresses on shear strain rates of high-viscosity oils. 1 - newtonian fluid; 2 - viscoplastic fluid; 3 - pseudoplastic; 4 - dilatant fluid; $\tau$ - shear stress; $\frac{d \gamma}{d t}-$ the rate of shear strain; $\tau_{0}$ - the initial shear stress for viscoplastic fluid; $\tau_{2}$ - the approximate value of the initial shear stress for viscoplastic fluid

Заметим, что время построения реологических кривых, как правило, не приводится. Из рисунка следует, что начальный период отклонения кривых 2-4 от аппроксимирующих прямых небольшой.

Известно, что для решения задач прогнозирования процессов фильтрации и выбора режимов 
эксплуатации скважин используются скорости фильтрации и градиенты давления. Ю.П. Желтовым в [5] приведена графическая зависимость скорости фильтрации дилатантной жидкости от градиента давления (рис. 3, б, кривая 4). Скорость деформации сдвига соответствует скорости фильтрации, касательное напряжение - градиенту давления. Зависимость скорости фильтрации от градиента давления для дилатантной жидкости нелинейная.

$$
\bar{v}=-\frac{k}{\mu}(|\operatorname{grad} p|)^{n}=-\frac{k}{\mu R} \operatorname{grad} p, \quad n<1,
$$

где $n$ - безразмерный параметр; $R$ - фактор сопротивления.

Сравнив (2) и (8), получим, что фактор сопротивления равен параметру Лагранжа.

По аналогии построим зависимости для ВПН, дилатантной жидкости и псевдопластиков в координатах градиент давления - скорость фильтрации (рис. 3).

Точки $\mathrm{A}_{2}, \mathrm{~A}_{3}$ и $\mathrm{A}_{4}$ соответствуют границам кривых 2-4, отделяющим малые значения текущих градиентов давления от больших значений текущих градиентов давления.

Аналитическая зависимость скорости фильтрации от градиента давления для псевдопластической жидкости аналогична (8), но безразмерный параметр $n>1$.

Для определения распределения давления псевдопластической и дилатантной жидкостей в зоне возмущения работающих скважин необходимо записать уравнение пьезопроводности В.Н. Щелкачева. Для этого следует подставить (8) в уравнение неразрывности (9)

$$
\frac{\partial}{\partial t}(m \rho)+\operatorname{div}(\rho v)=0
$$

где $m$ - коэффициент пористости; $\rho$ - плотность флюида.
С учётом сжимаемости флюида и коллектора получим нелинейное дифференциальное уравнение в частных производных относительно давления. Решение подобных уравнений вызывает определенные трудности. Для их устранения воспользуемся тем, что время работы эксплуатационных скважин измеряется сутками, месяцами. Отметим, что наибольшие текущие градиенты давления имеют место в призабойной зоне пласта (ПЗП), причем наибольшие значения градиентов давления конечны и не могут превышать величин, ведущих к разрушению коллектора. Области, где скорости фильтрации имеют малую величину, соответствуют удаленным зонам в окрестностях радиуса контура питания. Поэтому, исходя из физических соображений, предлагается нелинейные зависимости скоростей фильтрации от градиентов давления разбить на два участка, которые соответствуют ПЗП и удаленным зонам фильтрации. На рис. 3 пунктирными линиями изображены границы участков; параметры $g_{3}, g_{4}$ соответствуют начальным градиентам давлений для дилатантной и псевдопластической жидкостей. На первом участке, с большими градиентами давления (в том числе и в ПЗП), выполняется обобщенный закон Дарси (4). Классический закон Дарси для дилатантных и псевдопластиков, $g=0$, имеет место для удаленных зон, близких к контуру питания. А.Т. Горбуновым [1] отмечается, что в силу неоднородности коллектора в начале эксплуатации при малых градиентах давлений начинают работать прослои с более высокой проницаемостью, где влияние начального градиента $g$ незначительно. В процессе эксплуатации в работу подключаются прослои с меньшей проницаемостью, фильтрация в которых осуществляется по обобщенному закону Дарси (4).

После подстановки (4) в (9) уравнение пьезопроводности на первом участке для больших градиентов давления имеет вид:
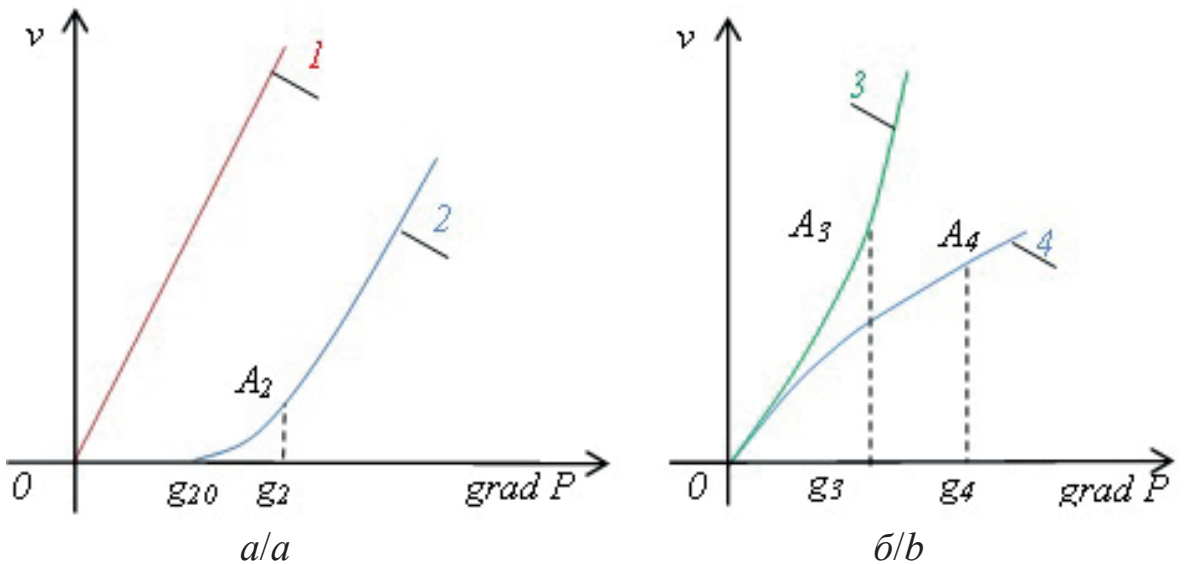

Pис. 3. Зависимости скоростей фильтраиии от градиентов давления высоковязких жидкостей: а) g $g_{20}, g_{2}-$ начальные градиенты давлений для ВПЖ, прямая линия 1 соответствует закону Дарси; кривая 2 - обобщеннолу закону Дарси; б) $g_{30}$, $g_{4}-$ начальные градиенты давлений для псевдопластиков (кривая 3) и дилатантной жидкости (кривая 4)

Fig. 3. Dependences of filtration rates on pressure gradients of high-viscosity liquids: a) $g_{20}, g_{2}$-initial pressure gradients for viscoplastic fluid, straight line 1 corresponds to the Darcy law; curve 2 - generalized Darcy law; $\sigma) g_{30}, g_{4}-$ initial pressure gradients for pseudoplastics (curve 3 ) and dilatant fluid (curve 4) 


$$
\frac{\partial p}{\partial t}=\chi \operatorname{div}(\operatorname{grad} p-g)
$$

а на втором участке для малых градиентов давления

$$
\frac{\partial p}{\partial t}=\chi \operatorname{div}(\operatorname{grad} p)
$$

где $\chi$ - коэффициент пьезопроводности.

Таким образом, используя результаты обработки гидродинамических исследований скважин на установившихся режимах, а при их отсутствии по лабораторным данным, можно определить начальные градиенты давления $g$ для дилатантных и псевдопластических жидкостей. Сшиванием решений уравнений (10) и (11) можно определить текущие границы участков 1 и 2.

\section{Оценка верхнего предела применимости закона Дарси}

Вернемся к оценке верхнего предела применимости закона Дарси. Параметр Лагранжа в этом случае $\lambda^{*}<1$. Из (3) следует, что коэффициент продуктивности $\eta^{*}(12)$ в этом случае превышает величину коэффициента продуктивности $\eta$, соответствующего закону Дарси, и равен

$$
\eta^{*}=\frac{Q}{\Delta p}=\frac{2 \pi k h}{\mu \lambda^{*}} \frac{1}{\ln \frac{R}{r_{c}}}=\frac{\eta}{\lambda^{*}} .
$$

Рассмотрим нелинейный закон Дарси для плоскорадиальной фильтрации в виде

$$
\frac{d p}{d r}=\frac{\mu(p)}{k} v-\frac{\rho(p)}{l} v^{2},
$$

где $\rho(p)$ - плотность флюида; $l$ - характерный параметр пористой среды (коэффициент макрошероховатости). Первое слагаемое правой части учитывает вязкостные силы, второе - инерционные. Если скорости фильтрации малы, то выполняется классический закон Дарси.

Плотность и вязкость флюида являются функциями давления, что существенно для фильтрации газов. При фильтрации жидкостей зависимостями плотности и вязкости от давления можно пренебречь. В случае плоскорадиальной фильтрации, подставляя скорость, выраженную через дебит в (13), после интегрирования получим известную зависимость депрессии от дебита для стационарной фильтрации

$$
\Delta p=A Q-B Q^{2},
$$

где $A=\frac{\mu}{2 \pi k h} \ln \frac{R}{r_{c}}, B=\frac{\rho}{(2 \pi h)^{2} l}\left(\frac{1}{r_{c}}-\frac{1}{R}\right) \approx \frac{\rho}{(2 \pi h)^{2} l r_{c}}$.

Параметр $A$ обратно пропорционален коэффициенту продуктивности. Из уравнения (14) при $\Delta p=0$ следует, что $Q=0$. Зависимость дебита от депрессии имеет вид

$$
Q=\frac{A}{2 B}\left(1-\sqrt{1-\frac{4 B \Delta p}{A^{2}}}\right) .
$$

Приравнивая (3) и (14), получим выражение для определения параметра Лагранжа $\lambda^{*}$

$$
\lambda^{*}=1-\frac{B}{A} Q<1 .
$$

Если параметр $\lambda^{*}$ известен, то из (16) можно определить параметры $B$ и l. Таким образом, установлена связь между коэффициентом макрошероховатости и параметром Лагранжа. Методика определения коэффициента макрошероховатости по результатам интерпретации гидродинамических исследований газовых скважин изложена в [39]. В литературе приводится зависимость, в которой коэффициент $l$ прямо пропорционален корню квадратному из коэффициента проницаемости. На наш взгляд такая зависимость не соответствует физической природе явления. Действительно, с увеличением проницаемости параметр $l$ возрастает, коэффициент $B$ в выражении (14) убывает, следовательно, нелинейная фильтрация при больших значениях коэффициента проницаемости превращается в линейную фильтрацию.

Для определения значения параметра Лагранжа и дебита при нарушении верхнего предела применимости закона Дарси для заданной депрессии рассмотрим следующий пример.

Пример 1. Дано $\mu=1 \mathrm{~m}$ Мас, $r_{c}=0,1 \mathrm{~m}, R=300 \mathrm{~m}$, $h=8 \mathrm{M}, l=0,7 \cdot 10^{-9} \mathrm{M}, \quad \Delta p=3 \mathrm{M \Pi а}, k=0,7 \cdot 10^{-12} \mathrm{M}^{2}$, $\rho=700 \kappa \Gamma / \mathrm{M}^{3}$.

Расчет проводится методом итераций:

1) по формулам находим коэффициенты $A, B$;

2) по формуле (15) определяем дебит на первом шаге;

3) из формулы (16) определим $\lambda^{*}$;

4) по параметру $\lambda^{*}$ из (3) определяем дебит;

5) повторяем процедуру, начиная с пункта 3.

Повторяя итерационный процесс, сходящийся на 6-й итерации, получим $Q=1769 \mathrm{~m}^{3} /$ сут, $\lambda^{*}=0,648$. Отметим, что $A$ и $B$ - постоянные величины, характеризующие фильтрационные свойства коллектора и физические свойства жидкости. В табл. 2 приведен расчет дебитов, определенных по формуле (15) и по формуле Дюпюи $\lambda^{*}=1$, для разных значений депрессий.

Таблица 2. Расчет дебитов для нелинейной фильтращии (15) и по формуле Дюпюи

Table 2. Calculation of flow rates for nonlinear filtering (15) and by the Dupuis formula

\begin{tabular}{|c|c|c|c|}
\hline $\begin{array}{c}\Delta p, \mathrm{M \Pi а} \\
\mathrm{MPa}\end{array}$ & $\begin{array}{c}Q, \mathrm{M}^{3} / \text { сут } \\
\mathrm{m}^{3} / \text { day }(15)\end{array}$ & $\begin{array}{c}Q, \mathrm{~m}^{3} / \text { сут, формула Дюпюи } \\
\text { Dupuis formula, } \mathrm{m}^{3} / \text { day }\end{array}$ & $\lambda^{*}$ \\
\hline 0 & 0 & 0 & 1,00 \\
\hline 0,5 & 197 & 189 & 0,960 \\
\hline 1 & 414 & 379 & 0,917 \\
\hline 2 & 935 & 759 & 0,812 \\
\hline 3 & 1769 & 1139 & 0,644 \\
\hline
\end{tabular}

Среднее значение параметра Лагранжа $\lambda_{c}^{*}=0,833$ (без первой строки).

Таким образом, с ростом депрессии для нелинейной фильтрации дебит больше, чем дебит, подсчитанный по формуле Дюпюи. Из формулы (15) 
видно, что максимальный дебит соответствует депрессии

$$
Q_{\max }=\frac{A}{2 B}, \quad \Delta p_{\max }=\frac{A^{2}}{4 B} .
$$

Если текущая депрессия больше $\Delta p_{\max }(17)$, то подкоренное выражение отрицательно и формула (15) не имеет физического смысла, но (любопытно), если взять модуль подкоренного выражения, то при $\Delta p=6,5$ МПа дебит будет равен нулю.

Если в формуле (13) второе слагаемое взять со знаком плюс:

$$
\frac{d p}{d r}=\frac{\mu(p)}{k} v+\frac{\rho(p)}{l} v^{2},
$$

то уравнение (14) примет вид

$$
\Delta p=A Q+B Q^{2} .
$$

Дебит и параметр Лагранжа определяются соотношениями

$$
Q=\frac{A}{2 B}\left(\sqrt{1+\frac{4 B \Delta p}{A^{2}}}-1\right), \quad \lambda^{*}=1+\frac{B}{A} Q>1 .
$$

Для данных рассмотренного выше примера 1 значения дебитов и параметра $\lambda^{*}$ приведены в табл. 3 .

Таблица 3. Расчет дебитов для нелинейной фильтрации (20) и по формуле Дюпюи

Table 3. Calculation of flow rates for nonlinear filtering (20) and by the Dupuis formula

\begin{tabular}{|c|c|c|c|}
\hline $\begin{array}{c}\Delta p, \mathrm{M \Pi а} \\
\mathrm{MPa}\end{array}$ & $\begin{array}{c}Q, \mathrm{~m}^{3} / \text { сут } \\
\mathrm{m}^{3} / \text { day }(20)\end{array}$ & $\begin{array}{c}Q, \mathrm{~m}^{3} / \text { сут, формула Дюпюи } \\
\mathrm{m}^{3} / \text { day, Dupuis formula }\end{array}$ & $\lambda^{*}$ \\
\hline 0 & 0 & 0 & 1,00 \\
\hline 1 & 354 & 379 & 1,07 \\
\hline 2 & 669 & 759 & 1,13 \\
\hline 3 & 955 & 1139 & 1,19 \\
\hline 4 & 1219 & 1518 & 1,25 \\
\hline 5 & 1465 & 1898 & 1,30 \\
\hline
\end{tabular}

Из табл. 3 видно, что $\lambda^{*}>1$ и дебит, подсчитанный по формуле Дюпюи, больше дебита, определенного по формуле (20). Среднее значение $\lambda_{c}^{*}=1,213$. Таким образом, уравнение (19) соответствует фильтрации флюида при нарушении нижнего предела применимости закона Дарси. Поэтому в левой части уравнения (19) следует учитывать предельное значение депрессии $\Delta p^{*}$. В результате получим

$$
\Delta p-\Delta p^{*}=A Q+B Q^{2} .
$$

При $\Delta p=\Delta p^{*}$ дебит $Q=0$. Величину $\Delta p^{*}$ можно определить по результатам обработки индикаторных диаграмм [38]. Заметим, что параметры в табл. 3 рассчитывались по данным примера 1 для большой проницаемости и маловязкой нефти. И тем не менее имеет место нарушение нижнего предела применимости закона Дарси. Действительно, из (3) и (21) имеем

$$
\lambda^{*}=1+\frac{B}{A} Q+\frac{\Delta p^{*}}{A Q}>1 .
$$

Таким образом, для описания стационарной фильтрации с нарушением нижнего предела применимости закона Дарси можно использовать две модели: 1) линейная - с учетом начального градиента давления, уравнения (5), (10); 2) нелинейная - уравнения (21), (22). Для иллюстрации рассмотрим низкопроницаемый коллектор со следующими данными, пример 2.

Пример 2. Дано $\mu=1 \mathrm{м}$ Мас, $r_{c}=0,1 \mathrm{~m}, R=100 \mathrm{M}$, $h=8 \mathrm{M}, \quad l=0,7 \cdot 10^{-9} \mathrm{M}, \quad k=7 \mathrm{~m} Д, \quad \rho=700 \kappa \Gamma / \mathrm{m}^{3}$, $\Delta p^{*}=0,1 \mathrm{M \Pi а}$.

В табл. 4 приведены результаты расчета дебитов по формулам (5) и (19), а также параметров Лагранжа по формулам (6), (20), в которой $\Delta p$ заменено на $\left(\Delta p-\Delta p^{*}\right)$, и $(22)$.

Таблица 4. Расчет дебитов и параметров Лагранжа для ВПН (5), (6) и для нелинейной фильтрации (18), (20), (22)

Table 4. Calculation of the rates and Lagrange parameters for viscoplastic fluid (5), (7) and for nonlinear filtration (18), (20), (22)

\begin{tabular}{|c|c|c|c|c|c|}
\hline $\begin{array}{c}\Delta p, \\
\mathrm{M \Pi a} / \mathrm{MPa}\end{array}$ & $\begin{array}{c}Q, \mathrm{~m}^{3} / \mathrm{cyT} \\
\mathrm{M}^{3} / \text { day }(5)\end{array}$ & $\lambda^{*}(6)$ & $\begin{array}{c}Q, \mathrm{~m}^{3} / \mathrm{cyT} \\
\mathrm{m}^{3} / \text { day }(20)\end{array}$ & $\lambda^{*}(20)$ & $\lambda^{*}(22)$ \\
\hline 0 & 0 & 1 & 0 & 1 & 1 \\
\hline 1 & 4 & 1,111 & 4 & 1,00001 & 1,10001 \\
\hline 2 & 8 & 1,053 & 9 & 1,00002 & 1,05002 \\
\hline 3 & 13 & 1,034 & 13 & 1,00003 & 1,03337 \\
\hline 4 & 17 & 1,025 & 18 & 1,00004 & 1,02504 \\
\hline 5 & 22 & 1,020 & 22 & 1,00005 & 1,02005 \\
\hline 6 & 26 & 1,017 & 26 & 1,00006 & 1,01673 \\
\hline
\end{tabular}

Дебиты, рассчитанные по формулам (5) и (20), практически совпадают. Параметр Лагранжа ведет себя по-разному. Для ВПН (6) и нелинейной фильтрации с учетом $\Delta p^{*}$, формула (22), $\lambda^{*}$ убывает с ростом давления. Если $\Delta p^{*}=0(20)$, то $\lambda^{*}$ возрастает. Причем $\lambda^{*}>1$, что свидетельствует о нарушении нижнего предела применимости закона Дарси. Если параметр $\lambda^{*}=1$, то дебит, подсчитанный по формуле Дюпюи, незначительно, но превышает дебиты, приведенные в таблице.

Уравнение (18) соответствует нелинейной стационарной фильтрации. Если решение уравнения (18) подставить в уравнение неразрывности, то получим нелинейное дифференциальное уравнение для определения давления при нестационарной фильтрации, решение которого, как уже ранее говорилось, вызывает определенные трудности. В связи с этим для вывода уравнения пьезопроводности, учитывающего особенности фильтрации в аномальных коллекторах, подставим скорость фильтрации (2) в уравнение неразрывности (9). В результате получим

$$
\begin{gathered}
\frac{\partial p}{\partial t}=\chi \operatorname{div}\left(\frac{1}{\lambda^{*}} \operatorname{grad} p\right) \approx \frac{\chi}{\lambda_{c}^{*}} \operatorname{div} \operatorname{grad} p= \\
=\chi_{c} \text { divgradp. }
\end{gathered}
$$


Здесь $\lambda_{c}^{*}$ - среднее значение параметра Лагранжа, определяемое из табл. 1,$2 ; \chi_{\tilde{n}}=\frac{\chi}{\lambda_{\tilde{n}}^{*}}-$ коэффициент пьезопроводности аномального коллектора. При $\lambda^{*}>1$ соответствует нарушению нижнего предела применимости закона Дарси, $\lambda^{*}<1$ соответствует нарушению верхнего предела применимости закона Дарси.

Отметим, что уравнение (23) можно разбить на два уравнения: для малых градиентов давления в удаленных зонах пласта и для больших градиентов в зоне ПЗП. Для каждого уравнения выбрать свое $\lambda^{*}$, затем решения «сшить».

\section{СПИСОК ЛИТЕРАТУРЫ}

1. Горбунов А.Т. Разработка аномальных нефтяных месторождений. - М.: Недра, 1981. - 237 с.

2. Огибалов П.М., Мирзаджанзаде А.Х. Нестационарные движения вязкопластичных сред. - М.: МГУ, 1977. - 372 с.

3. 0 возможном влиянии начального градиента на разработку многопластовых газовых и газоконденсатных месторождений при водонапорном режиме / А.Х. Мирзаджанзаде, Г.И. Баренблатт, В.М. Ентов, Ю.В. Желтов, Г.Д. Маргулов, Г.В. Рассохин, В.М. Рыжик // Известия высших учебных заведения. Нефть и газ. - 1970. - № 1. - С. 39-45.

4. Гиматутдинов Ш.К., Ширковский А.И. Физика нефтяного и газового пласта. - М.: Изд-во «Недра», 1982. - 311 с.

5. Желтов Ю.П. Разработка нефтяных месторождений. - М.: ОА0 «Недра», 1998. - 365 с.

6. Физические модели вытеснения вязкопластичных нефтей В.А. Коротенко, С.И. Грачев, Н.П. Кушакова, Р.Р. Сабитов // Нефтепромысловое дело. - 2014. - № 5. - С. 5-10.

7. Особенности вытеснения вязкопластичной нефти водой В.А. Коротенко, Н.П. Кушакова, И.В. Сидоров, Р.Р. Сабитов // Электронный научный журнал «Нефтегазовое дело». - 2013. № 5. - С. 190-196.

8. Медведский Р.И., Севастьянов А.А. Оценка извлекаемых запасов нефти и прогноз уровней добычи по промысловым данным. - СПб.: Недра, 2004. - 192 с.

9. Севастьянов А.А., Карнаухов К.Н., Коровин К.В. Обоснование технологических решений для повышения эффективности выработки запасов нефти // Новые информационные технологии в нефтегазовой отрасли и образовании: Труды II Международной науч.-тех. конференции. - Тюмень, 2006. - С. 178-182.

10. Development of ultra low permeability reservoirs / I.S. Afanasiev, V.A. Baikov, A.V. Kolonskikh, A.I. Fedorov, V.V. Maltsev // Нефтяное хозяйство. - 2014. - № 5. - С. 82-86.

11. Алишаев М.Г., Розенберг М.Д., Теслюк Е.В. Неизотермическая фильтрация при разработке нефтяных месторождений. М.: Недра, 1985. - 275 с.

12. Модели фильтрации вязкопластических флюидов и критические (начальные) градиенты давления // А.К. Ягафаров, Н.П. Кушакова, В.А. Коротенко, А.Н. Сумин // Наука и ТЭК. - 2012. - № 5. - С. 18-20.

13. Телков А.П., Грачев С.И. Гидромеханика пласта применительно к прикладным задачам разработки нефтяных и газовых месторождений. В 2 ч. Ч. 1. - Тюмень: ТюмГНГУ, 2009. - 240 с.

14. К вопросу о вытеснении нефти из аномальных коллекторов С.И. Грачев, В.А. Коротенко, Н.П. Кушакова, О.П. Зотова // Успехи современного естествознания. - 2016. - № 10. C. $114-118$

15. Шпильман А.В., Коровин К.В., Савранская М.П. Перспективы освоения ТРИЗ В ХМАО-ЮГРЕ // НЕФТЬГАЗТЭК: ТрудЫ

\section{Заключение}

Установлен критерий применимости закона Дарси для исследований процессов фильтрации и вытеснения нефти водой (табл. 1) при значениях параметра Лагранжа более единицы. Для описания фильтрации флюидов в аномальных коллектоpax следует рассматривать две модели: линейную - с учетом начального градиента давления, уравнения (5), (10); и нелинейную - уравнение (21). Выбор оптимальной модели осуществляется посредством сравнения фактических промысловых и расчетных значений, с учетом имеющихся результатов гидродинамических и лабораторных исследований.

6 Тюменского международного инновационного форума. - Тюмень, 2015. - С. 461-464.

16. Севастьянов А.А., Коровин К.В., Карнаухов А.Н. Выявление особенностей механизма выработки запасов нефти по месторождениям Ханты-Мансийского автономного округа // Известия высших учебных заведений. Нефть и газ. -2007 . - № 3. C. $32-38$.

17. Брехунцов А.М., Телков А.П., Федорцов В.К. Развитие теории фильтрации жидкости и газа к горизонтальным стволам скважин. - Тюмень: ОАО «СибНАЦ», 2004. - 290 с.

18. Телков А.П. Особенности разработки нефтегазовых месторождений. - Тюмень: ТюмГНГУ, 2001. - 482 c.

19. Revisiting the development of oil deposits with low permeability reservoirs / S.N. Zakirov, A.A. Barenbaum, E.S. Zakirov, I.M. Indrupskiy, V.A. Serebryakov, D.S. Klimov // Indian Journal of Science and Technology. - 2016. - № 42. - P. 104219. DOI: 10.17485/ijst/2016/v9i42/104219, November 2016.

20. Effect of chemical additives on dynamic capillary pressure during waterflooding in low permeability reservoirs / H. Li, Y. Li, K. Wang, H. Luo, S. Chen, J. Guo // Energy and Fuels. - 2016. № 9. - P. 7082-7093.

21. Effect of quadratic pressure gradient term on a one-dimensional moving boundary problem based on modified Darcy's law / W. Liu, Y. Liu, J. Yao, Z. Chen // Acta Mechanica Sinica. 2016. - № 1. - P. 38-53.

22. Cao Y., Yuan G., Xi K. Preliminary discussion of improvement experiments on strong sensitivity-low permeability // Xinan Shiyou Daxue Xuebao. - 2012. - № 1. - P. 90-96.

23. A new method for testing relative permeability of ultra-low permeability reservoirs / Z. Yang, Z. Li, X. Wang, W. Shan // Shiyou Xuebao. - 2010. - № 4. - P. 629-632.

24. Nonlinear flow numerical simulation of an ultra-low permeability reservoir / R.-Z. Yu, Z.-M. Yang, Q. Lei, Y.-N. Bian // Chinese Physics Letters. - 2010. - № 7. - P. 074702.

25. Dynamic effect of capillary pressure in low permeability reservoirs / S. Tian, G. Lei, S. He, L. Yang // Petroleum Exploration and Development. - 2012. - № 3. - P. 405-411.

26. Dynamic effect of capillary pressure in low permeability reservoirs / S. Tian, G. Lei, S. He, L. Yang // Shiyou Kantan Yu Kaifa. - 2012. - № 3. - P. 378-384.

27. He J.G., Song K.P., Yang J. Study on experiment of advance water injection an example from low permeability oil reservoir of Fuyu oil reservoir in an oil field // Science Technology and Engineering. - 2014. - V. 14 (11). - P. 181-183.

28. Hu Z.M., Ba Z.B., Xiong W. Analysis of micro-pore structure in low permeability reservoirs // Journal of A Petroleum Institute. 2006. - V. 30 (3). - P. 51-53.

29. Wang W., Peng H., Li S. Opening mechanism of dynamic fractures caused by water injection and effective adjustment in low per- 
meability reservoirs, Daqing oilfield in Songliao basin // 0il \& gas geology. - 2015. - V. $36(5) .-$ P. 842-847.

30. Vishnudas R., Chaudhuri A. A comprehensive numerical study of immiscible and miscible viscous fingers during chemical enhanced oil recovery // Fuel. - 2016. - V. 194. - P. 480-490.

31. Loh W.L., Premanadhan V.K. Experimental investigation of viscous oil-water flows in pipeline // Journal of Petroleum Science and Engineering. - 2016. - V. 147. - P. 89-97.

32. Investigation on flow patterns and pressure drops of highly viscous crude oil-water flows in a horizontal pipe / J. Jiaqiang, S. Jie, T. Jiatong, H. Min, X. Tao // Experimental Thermal and Fluid Science. - 2016. - V. 72. - P. 88-96.

33. Maheswaran R., Sunil J. Effect of nano sized garnet particles dispersion on the viscous behavior of extreme pressure lubricant oil // Journal of Molecular Liquids. - 2016. - 223. - P. 643-651.

34. Transport and removal of a solvent in porous media in the presence of bitumen, a highly viscous solute / S. Panda, K. Pal, S. Merzara, M.R. Gray, P. Choi // Chemical Engineering Science. 2017. - V. 165. - P. 229-239.

35. Hydrodynamic study of multiphase flow transport of highly viscous foamy fluids / A. Brito, R. Cabello, N. Guzman, L. Marcano,
J. Trujillo // Journal of Petroleum Science and Engineering. 2015. - V. 135. - P. 367-374.

36. Dong T., Cao S., Xu G. Highly porous oil sorbent based on hollow fibers as the interceptor for oil on static and running water // Journal of Hazardous Materials. - 2015. - V. 305. - P. 1-7.

37. Arabloo M., Shokrollahi A., Ghazanfari M.H., Rashtchian D. Characterization of viscous fingering during displacements of low tension natural surfactant in fractured multi-layered heavy oil systems // Chemical Engineering Research and Design. 2015. - V. 72. - P. 23-34.

38. Коротенко В.А., Ягафаров А.К., Сумин А.Н. Определение начального градиента давления по результатам гидродинамических исследований скважин // Известия вузов: Нефть и газ. 2012. - № 6. - C. 55-58.

39. Пространственная фильтрация и прикладные задачи разработки нефтегазоконденсатных месторождений и нефтегазодобычи / А.П. Телков, С.И. Грачев, Е.И. Гаврилов, Т.Л. Краснова. - Тюмень: 00 НИПИКБС-Т, 2001. - 460 с.

Поступила 15.11.2018 2.

\section{Информация об авторах}

Грачев С.И., доктор технических наук, профессор, заведующий кафедрой разработки и эксплуатации нефтяных и газовых месторождений Института геологии и нефтегазодобычи Тюменского индустриального университета.

Коротенко В.A., кандидат технических наук, доцент кафедры разработки и эксплуатации нефтяных и газовых месторождений Института геологии и нефтегазодобычи Тюменского индустриального университета.

Кушакова Н.П., кандидат технических наук, доцент кафедры автомобильного транспорта, строительных и дорожных машин Строительного института Тюменского индустриального университета.

Кряквин A.Б., кандидат технических наук, доцент кафедры разработки и эксплуатации нефтяных и газовых месторождений Института геологии и нефтегазодобычи Тюменского индустриального университета.

Зотова О.П., ассистент кафедры разработки и эксплуатации нефтяных и газовых месторождений Института геологии и нефтегазодобычи Тюменского индустриального университета. 
UDC 622.276

\title{
LIQUID FILTRATION IN ANOMALOUS COLLECTORS
}

\author{
Sergey I. Grachev', \\ grachevsi@mail.ru
Valentin A. Korotenko', grachevsi@mail.ru

\section{Nelli P. Kushakova',}

kushakovanp@tyuiu.ru

\author{
Aleksandr B. Kryakvin', \\ grachevsi@mail.ru \\ Olga P. Zotova', \\ zotovaop@tsogu.ru \\ ${ }^{1}$ Tyumen Industrial University, \\ 38, Volodarsky street, Tyumen, 625000, Russia.
}

The relevance of the research is caused by the need of development of Jurassic and Achimov reservoirs with low filtration properties and viscoplastic oil deposits. Classic Darcy law can not be applied to these reservoirs. So to predict development indicators and to calculate well production potential the filtration and oil displacement features should be considered.

The main aim of the research is to find the relation between Lagrange parameter and initial pressure gradient, to identify values of Lagrange parameter when Darcy law can not be applied. The aim of the research is to explain as well the hydrodynamic modeling methods (developed for liquid filtration) application to research of fluid flow in anomalous reservoirs.

Objects: high-permeability reservoirs with high-viscosity (viscoplastic) oil; low-permeability Jurassic and Achim layers.

Methods: interpretation of laboratory core researches; physical modeling of fluid filtration in anomalous reservoirs.

Results. The deviation from Darcy law for liquid filtration in anomalous reservoirs is defined by Lagrange parameter calculation. Method of determination of lower limit of Darcy Law applicability is proposed. For the case of Darcy Law violation two models are proposed: with initial pressure gradient and nonlinear model. Nonlinear model is considered for the case of upper limit of Darcy law vialation. Lagrange parameter calculation examples are considered for upper and lower limits of Darcy's Law vialation. It is proposed to use system of piezoconductivity equations instead of nonlinear equations for dilatant and pseudoplastic fluids filtration. It is shown that methods for solving hydrodynamic filtration and oil displacement problems are applicable to both viscoplastic oils and low-permeability reservoirs.

\section{Key words:}

Anomalous collectors, limits of applicability of the Darcy law, initial pressure gradient, mobility coefficient, Lagrange parameter.

\section{REFERENCES}

1. Gorbunov A.T. Razrabotka anomalnykh neftyanykh mestorozhdeniy [Development of anomalous oil deposits]. Moscow, Nedra Publ., 1981. $237 \mathrm{p}$.

2. Ogibalov P.M., Mirzadzhanzade A.H. Nestatsionarnye dvizheniya vyazkoplastichnykh sred [Nonstationary motions of viscoplastic media]. Moscow, MGU Publ., 1977. 372 p.

3. Mirzadzhanzade A.H., Barenblatt G.I., Entov V.M., Zheltov Yu.V., Margulov G.D., Rassokhin G.V., Ryzhik V.M. 0 vozmozhnom vliyanii nachalnogo gradienta na razrabotku mnogoplastovykh gazovykh i gazokondensatnykh mestorozhdeniy pri vodonapornom rezhime [0n possible influence of the initial gradient on development of multi-layer gas and gas-condensate fields in the water-pressure regime]. Izvestiya vysshikh uchebnykh zavedeniy. Neft i gaz, 1970, no. 1, pp. 39-45.

4. Gimatutdinov S.K., Shirkovskiy A.I. Fizika neftyanogo $i$ gazovo go plasta [Physics of oil and gas reservoir]. Moscow, Nedra Publ., 1982. $311 \mathrm{p}$.

5. Zheltov I.P. Razrabotka neftyanykh mestorozhdeniy [Development of oil fields]. Moscow, Nedra Publ., 1998. 365 p.

6. Korotenko V.A., Grachev S.I., Kushakova N.P., Sabitov R.R. Fizicheskie modeli vytesneniya vyazkoplastichnykh neftey [Physical models of viscous-plastic oils displacement]. Neftepromyslovoe delo, 2014, no. 5, pp. 5-10.
7. Korotenko V.A., Kushakova N.P., Sidorov I.V., Sabitov R.R. Features of viscoplastic oil replacement by water. Elektronnyi nauchnyy zhurnal "Neftegazovoe delo», 2013, no. 5, pp. 190-196. In Rus.

8. Medvedskiy R.I., Sevastyanov A.A. Otsenka izulekaemykh zapasou nefti i prognoz urouney dobychi po promyslovym dannym [Estimation of recoverable oil reserves and forecast of production levels from field data]. St-Petersburg, Nedra Publ., 2004. 192 p.

9. Sevastyanov A.A., Karnaukhov K.N., Korovin K.V. Obosnovanie tekhnologicheskikh resheniy dlya povysheniya effektivnosti vyrabotki zapasov nefti [Substantiation of technological solutions for increasing the efficiency of producing oil reserves]. Trudy II Mezhdunarodnoy nauchno-tekhnicheskoy konferentsii. Novye informatsionnye tekhnologii $v$ neftegazovoy otrasli $i$ obrazovanii [Proc. 2 Int. scientific-tech. conf. New Information Technologies in the Oil and Gas Industry and Education]. Tyumen, 2006. pp. 178-182.

10. Afanasiev I.S., Baikov V.A., Kolonskikh A.V., Fedorov A.I., Maltsev V.V. Development of ultra low-permeability oil reservoirs. Oil industry, 2014, no. 5, pp. 82-86. In Rus.

11. Alishaev M.G., Rozenberg M.D., Teslyuk E.V. Neizotermicheskaya filtratsiya pri razrabotke neftyanykh mestorozhdeniy [Nonisothermal filtration in development of oil fields]. Moscow, Nedra Publ., 1985. 275 p. 
12. Yagafarov A.K., Kushakova N.P., Korotenko V.A., Sumin A.N. Modeli filtratsii vyazkoplasticheskikh flyuidov i kriticheskie (nachalnye) gradienty davleniya [Models of filtration of viscoplastic fluids and critical (initial) pressure gradients]. Nauka i TEK, 2012, no. 5, pp. 18-20.

13. Telkov A.P., Grachev S.I. Gidromekhanika plasta primenitelno $k$ prikladnym zadacham razrabotki neftyanykh $i$ gazovykh mestorozhdeniy [Hydrodynamics of the reservoir in relation to application to develop oil and gas fields]. Tyumen, TyumGNGU Publ., 2009. $240 \mathrm{p}$.

14. Grachev S.I., Korotenko V.A., Kushakova N.P., Zotova O.P. The question of the displacement of oil from the anomalous collectors. Uspekhi sovremennogo estestvoznaniya, 2016, no. 10, p. 114-118. In Rus.

15. Shpilman A.V., Korovin K.V., Savranskaya M.P. Perspektivy osvoeniya TRIZ V HMA0-IuGRE [Perspectives of TDF development in KhMA0-Iugra]. Trudy 6 Tyumenskogo mezhdunarodnogo innovatsionnogo foruma. NEFTGAZTEK [Proc. $6^{\text {th }}$ Tyumen Int. Innovation Forum NEFTGAZTEK.]. Tyumen, 2015. pp. 461-464.

16. Sevastyanov A.A., Korovin K.V., Karnaukhov A.N. Vyyavlenie osobennostey mekhanizma vyrabotki zapasov nefti po mestorozhdeniyam Khanty-Mansiyskogo avtonomnogo okruga [Identification of features of the mechanism of oil reserves development in the fields of Khanty-Mansiysk Autonomous 0krug]. Izvestiya vysshikh uchebnykh zavedeniy. Neft i gaz, 2007, no. 3. pp. 32-38.

17. Brekhuntsov A.M., Telkov A.P., Fedortsov V.K. Razvitie teorii filtratsii zhidkosti i gaza $k$ gorizontalnym stvolam skvazhin [Development of the theory of fluid and gas filtration to horizontal wellbores]. Tyumen, SibNAC Publ., 2004. 290 p.

18. Telkov A.P. Osobennosti razrabotki neftegazouykh mestorozhdeniy [Features of development of oil and gas fields]. Tyumen, TyumGNGU Publ., 2001. $482 \mathrm{p}$.

19. Zakirov S.N., Barenbaum A.A., Zakirov E.S., Indrupskiy I.M., Serebryakov V.A., Klimov D.S. Revisiting the development of oil deposits with low permeability reservoirs. Indian Journal of Science and Technology, 2016, no. 42, pp. 104219.

20. Li H., Li Y., Wang K., Luo H., Chen S., Guo J. Effect of chemical additives on dynamic capillary pressure during waterflooding in low permeability reservoirs. Energy and Fuels, 2016, no. 9, pp. 7082-7093.

21. Liu W., Liu Y., Yao J., Chen Z. Effect of quadratic pressure gradient term on a one-dimensional moving boundary problem based on modified Dacys law. Acta Mechanica Sinica, 2016, no. 1 (32), pp. 38-53.

22. Cao Y., Yuan G., Xi K. Preliminary discussion of improvement experiments on strong sensitivity-low permeability. Xinan Shiyou Daxue Xuebao, 2012, no. 1, pp. 90-96.

23. Yang Z., Li Z., Wang X., Shan W. A new method for testing relative permeability of ultra-low permeability reservoirs. Shiyou Xuebao, 2010, no. 4, pp. 629-632.

24. Yu R.-Z., Yang Z.-M., Lei Q., Bian Y.-N. Nonlinear flow numerical simulation of an ultra-low permeability reservoir. Chinese Physics Letters, 2010, no. 7, pp. 074702.

25. Tian S., Lei G., He S., Yang L. Dynamic effect of capillary pressure in low permeability reservoirs. Petroleum Exploration and Development, 2012, no. 3, pp. 405-411.
26. Tian S., Lei G., He S., Yang L. Dynamic effect of capillary pressure in low permeability reservoirs. Shiyou Kantan Yu Kaifa, 2012, no. 3, pp. 378-384.

27. He J.G., Song K.P., Yang J. Study on experiment of advance water injection an example from low permeability oil reservoir of Fuyu oil reservoir in an oil field. Science Technology and Engineering, 2014, no. 14 (11), pp. 181-183.

28. Hu Z.M., Ba Z.B., Xiong W. Analysis of micro-pore structure in low permeability reservoirs. Journal of a Petroleum Institute, 2006, vol. 30 (3), pp. 51-53.

29. Wang W., Peng H., Li S. Opening mechanism of dynamic fractures caused by water injection and effective adjustment in low permeability reservoirs, Daqing oilfield in Songliao basin. Oil \& gas geology, 2015, no. 36 (5), pp. 842-847.

30. Vishnudas R., Chaudhuri A. A comprehensive numerical study of immiscible and miscible viscous fingers during chemical enhanced oil recovery. Fuel, 2016, no. 194, pp. 480-490.

31. Loh W.L., Premanadhan V.K. Experimental investigation of viscous oil-water flows in pipeline. Journal of Petroleum Science and Engineerin, 2016, no. 147, pp. 89-97.

32. Jiaqiang J., Jie S., Jiatong T., Min H., Tao X. Investigation on flow patterns and pressure drops of highly viscous crude oil-water flows in a horizontal pipe. Experimental Thermal and Fluid Science, 2016, no. 72, p. 88-96.

33. Maheswaran R., Sunil J. Effect of nano sized garnet particles dispersion on the viscous behavior of extreme pressure lubricant oil. Journal of Molecular Liquids, 2016, no. 223, pp. 643-651.

34. Panda S., Pal K., Merzara S., Gray M.R., Choi P. Transport and removal of a solvent in porous media in the presence of bitumen, a highly viscous solute. Chemical Engineering Science, 2017, no. 165 , p. 229-239.

35. Brito A., Cabello R., Guzman N., Marcano L., Trujillo J. Hydrodynamic study of multiphase flow transport of highly viscous foamy fluids. Journal of Petroleum Science and Engineering, 2015, no. 135, p. $367-374$.

36. Dong T., Cao S., Xu G. Highly porous oil sorbent based on hollow fibers as the interceptor for oil on static and running water. Journal of Hazardous Materials, 2015, no. 305, pp. 1-7.

37. Arabloo M., Shokrollahi A., Ghazanfari M.H., Rashtchian D. Characterization of viscous fingering during displacements of low tension natural surfactant in fractured multi-layered heavy oil systems. Chemical Engineering Research and Design, 2015, no. 72 , pp. 23-34.

38. Korotenko V.A., Yagafarov A.K., Sumin A.N. Defining the initial pressure gradient based on the results of hydrodynamic studies of wells. Izvestiya vuzov: Neft i gaz, 2012, no. 6, pp. 55-58. In Rus.

39. Telkov A.P., Grachev S.I., Gavrilov E.I., Krasnova T.L. Prostranstvennaya filtratsiya i prikladnye zadachi razrabotki neftegazokondensatnykh mestorozhdeniy i neftegazodobychi [Spatial filtration and applied problems of development of oil and gas condensate fields and oil and gas production]. Tyumen, NIPIKBS-T Publ., 2001. $460 \mathrm{p}$.

Received: 15 November 2018.

\section{Information about the authors}

Sergey I. Grachev, Dr. Sc., professor, head of the department of exploration and production of oil and gas fields, Tyumen Industrial University.

Valentin A. Korotenko, Cand. Sc., associate professor, Tyumen Industrial University.

Nelli P. Kushakova, Cand. Sc., associate professor, Tyumen Industrial University.

Aleksandr B. Kryakvin, Cand. Sc., associate professor, Tyumen Industrial University.

Olga P. Zotova, assistant, Tyumen Industrial University. 